\title{
The electron loss peak in ion-atom collisions: a probe for the projectile or for the target atomic structure?
}

\author{
D H Jakubassa-Amundsen
}

Physics Section, University of Munich, 8046 Garching, Federal Republic of Germany

Received 28 September 1992, in final form 4 February 1993

\begin{abstract}
A double peak structure is predicted in the spectrum of electrons which are emitted from the projectile in the backward direction in collision with heavy target atoms. The calculations have been performed within the electron impact approximation and the strong potential second Born theory for hydrogen-like light projectiles colliding with Ar and $\mathrm{Xe}$. The structure in the electron loss peak is only present in a certain range of intermediate collision velocities. It is sensitive to the electronic density distribution in the target and may therefore serve as a tool for investigating atomic fields in gaseous and solid targets.
\end{abstract}

An accurate knowledge of atomic electron loss cross sections is important for equilibration processes of beams of charged particles traversing solid targets, because they affect the projectile charge distribution and hence the stopping power. Considerably more information than in total loss cross sections is contained in the energy and angular distribution of the emitted electrons, which allows for a detailed spectroscopy of the collision partners. For energetic projectiles colliding with light targets, it is well established and confirmed by the first-order Born approximation (Drepper and Briggs 1976) that the shape of the electron loss peak which appears near $E_{\text {loss }}=\frac{1}{2} v^{2}+\varepsilon_{i}^{\mathrm{P}}$ (for emission angles above $\sim 10^{\circ}$ ) reflects the Compton profile of the initial bound projectile electron, while its location is determined by the collision velocity $v$ and the initial binding energy $-\varepsilon_{?}^{\text {P }}$.

The breakdown of a first-order approach for electron ejection by a heavy collision partner has recently been demonstrated experimentally by Kelbch et al (1989) and Richard et al (1990). Investigating the binary encounter peak for target ionization, they found that not only the scaling with the square of the projectile effective charge is violated when partly stripped heavy projectiles are used (Richard et al 1990), but also that instead of the familiar shape of the peak, a double peak structure appears in a narrow range of emission angles (Kelbch et al 1989, Reinhold et al 1991). These features indicate that the active target electron is strongly influenced by the ionic field of the projectile. In fact, the experimental results can be explained when target ionization by a heavy perturber is described in terms of quasielastic scattering of the active electron by the perturber potential (Reinhold et al 1991, Shingal et al 1990).

The binary encounter peak which exists only at emission angles below $90^{\circ}$ corresponds, however, to the electron loss peak at backward angles in the reversed collision system (Drepper and Briggs 1976). With the advent of new coincidence techniques (DuBois and Manson 1986) which allow for a separation of electron loss from pure target ionization by means of detecting the electron simultaneously with the charge 
state of the transmitted projectile, electron loss is likewise well suited for investigating the effects of a strong perturber potential. Coincidence measurements of the backwardangle loss spectra from $\mathrm{H}$ and $\mathrm{He}$ in collision with heavy targets like Ar have up to now only been carried out at rather high collision velocities (DuBois and Manson 1990, Kuzel et al 1992) where peak structures are expected to be weak. Since the loss peak is present at all velocities one might, however, easily run the experiments at much lower velocities in order to see stronger effects of the perturber. This is an advantage compared to binary encounter peak investigations, because that peak disappears in the background at low collision velocities.

For electron loss induced by heavy targets, the inadequacy of perturbation theory has been known for nearly two decades (Walters 1975). Approaches like the classical elastic scattering model (Burch et al 1973) as well as the quantum mechanical electron impact approximation (Jakubassa 1980) and the 'impulse approximation' of Hartley and Walters (1987) have been applied to explain the experimental data. In the present work, the electron impact approximation (EIA) and the strong potential second Born theory (sB2) are used. Briefly, in the EIA the final electronic state is taken as a scattering eigenstate to the target atomic potential, and in the transition operator, only the zeroth-order term of an expansion in terms of the weak field (i.e. the projectile core field) is retained (Jakubassa-Amundsen 1992). This zeroth-order term is the leading order term for charge transfer to the continuum. Its classical correspondence is just the elastic scattering model which assumes that the active electron is quasifree and scatters elastically by the target potential. This is in contrast to charge transfer to bound states where the zeroth-order term is classically forbidden, and hence a first-order theory in the weak field (the strong potential Born approximation (JakubassaAmundsen and Amundsen 1980, Macek and Shakeshaft 1980) or its on-shell version, the impulse approximation) is needed for a proper description. The doubly differential cross section for electron loss is obtained from folding the cross section for elastic scattering by the target field $\mathrm{d}_{2} \mathrm{~d} \sigma_{\mathrm{e}} / \mathrm{d} \Omega$, with the momentum-space density $\left|\varphi_{1}^{\mathrm{P}}\right|^{2}$ of the initial electronic bound state (in atomic units, $\hbar=m=e=1$ )

$$
\frac{\mathrm{d}^{2} \sigma^{\mathrm{EIA}}}{\mathrm{d} E_{j} \mathrm{~d} \Omega_{f}}=\frac{k_{f}}{v} \int \mathrm{d} \boldsymbol{k}_{t}\left|\varphi_{i}^{\mathrm{P}}\left(k_{i}\right)\right|^{2} \frac{\mathrm{d} \sigma_{\mathrm{c}}}{\mathrm{d} \Omega}\left(k_{i}+v, k_{f}\right) \delta\left(E_{f}-\varepsilon_{i}^{\mathrm{P}}-\frac{1}{2} v^{2}-v k_{1}\right)
$$

in the case of an inert target. The energy and solid angle of the ejected electron in the target frame of reference is denoted by $E_{f}=\frac{1}{2} k_{f}^{2}$ and $\Omega_{j}$, respectively. The appearance of the initial $\left(\boldsymbol{k}_{1}+\boldsymbol{v}\right)$ and final $\left(\boldsymbol{k}_{f}\right)$ momentum of the scattering electron in $\mathrm{d} \sigma_{\mathrm{e}} / \mathrm{d} \Omega$ (with $\left|k_{i}+v\right| \neq k_{f}$ in general) indicates that the process actually is off-shell. In the on-shell formula (1), inelasticity is accounted for by calculating scattering momentum $k$ and angle $\theta$ according to the prescription $k=\max \left(\left|k_{i}+v\right|, k_{f}\right)$ and $\sin \theta / 2=$ $\left|\boldsymbol{k}_{i}+\boldsymbol{v}-\boldsymbol{k}_{f}\right| / 2 k$ (Hartley and Walters 1987).

In the SB2 theory, a projectile eigenstate is chosen for the outgoing electron. Hence, SB2 is the second-order Born theory for direct ionization with the electronic propagation in the strong potential (i.e. the target atomic field). For an inert target, the transition probability from the initial $\left(\psi_{l}^{\mathrm{P}}\right)$ to the final $\left(\psi_{f}^{\mathrm{p}}\right)$ electronic state reads in the semiclassical formulation

$$
a_{f i}^{\mathrm{SB} 2}=-\mathrm{i} \int \mathrm{d} t\left\langle\psi_{f}^{\mathrm{P}}\left|V_{\mathrm{T}}+V_{\mathrm{T}} G_{\mathrm{T}} V_{\mathrm{T}}\right| \psi_{l}^{\mathrm{P}}\right\rangle
$$

where the propagator $G_{T}=\left(i \partial_{t}-T-V_{T}+i \varepsilon\right)^{-1}$ has been introduced, with $T$ the kinetic energy of the active electron and $V_{\mathrm{T}}$ the target field. Restricting the intermediate 
electronic states to on-shell target scattering states and introducing a full peaking approximation, one arrives at the peaked strong potential second Born expression for the doubly differential loss cross section introduced by Hartley and Walters (1987) as the 'impulse approximation'

$\frac{\mathrm{d}^{2} \sigma^{\mathrm{PSB} 2}}{\mathrm{~d} E_{f} \mathrm{~d} \Omega_{f}}=\frac{k_{f}}{v} \int \mathrm{d} \boldsymbol{k}_{t}\left|\left\langle\psi_{f}^{\mathrm{P}}(\boldsymbol{r})\left|\exp \left(-\mathrm{i} k_{i} \boldsymbol{r}\right)\right| \psi_{i}^{\mathrm{P}}(\boldsymbol{r})\right\rangle\right|^{2} \frac{\mathrm{d} \sigma_{\mathrm{e}}}{\mathrm{d} \Omega}\left(\boldsymbol{k}_{i}+\boldsymbol{k}_{f}, \boldsymbol{k}_{j}\right) \delta\left(\varepsilon_{f}^{\mathrm{P}}-\varepsilon_{\mathrm{f}}^{\mathrm{P}}-\boldsymbol{v} \boldsymbol{k}_{\mathrm{r}}\right)$

with $\varepsilon_{f}^{\mathrm{P}}=\frac{1}{2}\left(\boldsymbol{k}_{f}-\boldsymbol{v}\right)^{2}$. Although equations (1) and (3) are much alike, the EIA and the peaked SB2 are conceptionally different theories. Neglecting the influence of the projectile core field on the outgoing electron (i.e. casting (3) into (1)) would imply a change in the boundary conditions.

The investigation of electron loss is complicated by the fact that the target will be excited simultaneously with the ejection of a projectile electron (Briggs and Drepper 1978), because the momentum transfer required to ionize the loosely bound target valence electrons is comparable or even smaller than the momentum absorbed by the projectile electron. Hence, a so-called doubly inelastic (DI) contribution has to be added to the singly inelastic (i.e. inert-target) loss cross sections discussed above, and it has recently been pointed out that a second-order theory is required for its appropriate description (Jakubassa-Amundsen 1992, Wang et al 1992). For backward emission angles, the dominant DI contribution arises from two independent interactions, one between the active projectile electron and the atomic target (causing electron loss), the other between a target electron and the projectile core. Using the EIA prescription for the projectile electron and the first-order Born theory for target ionization, the DI contribution to the doubly differential loss cross section can be approximated as (Jakubassa-Amundsen 1992)

$$
\begin{aligned}
\frac{\mathrm{d}^{2} \sigma^{\mathrm{DI}}}{\mathrm{d} E_{f} \mathrm{~d} \Omega_{f}}=\frac{k_{f} Z_{\mathrm{P}}^{2}}{4 \pi^{4} v} \sum_{n=1}^{N_{\mathrm{T}}} \int \mathrm{d} \kappa_{\mathrm{T}} \int \mathrm{d} k_{\imath} \delta\left(\Delta E_{f n}^{\mathrm{T}}+E_{f}-\varepsilon_{i}^{\mathrm{P}}-\frac{1}{2} v^{2}-v k_{l}\right) \\
\quad \times\left|\int \frac{\mathrm{d} s}{s^{2}} \varphi_{i}^{\mathrm{P}}\left(k_{\mathrm{r}}-s\right) \frac{1}{\Delta E_{j n}^{\mathrm{T}}-s v+\mathrm{i} \varepsilon} f_{\mathrm{e}}\left(\boldsymbol{k}_{i}+v-s, k_{f}\right)\left\langle\varphi_{f}^{\mathrm{T}}(\boldsymbol{r})\left|\mathrm{e}^{\mathrm{i} s r}\right| \varphi_{n}^{\mathrm{T}}(\boldsymbol{r})\right\rangle\right|^{2}
\end{aligned}
$$

where $Z_{\mathrm{p}}$ is the projectile charge, $N_{\Upsilon}$ the number of target electrons, $\varphi_{n}^{\mathrm{T}}$ their initial bound state, $\varphi_{f}^{\mathrm{T}}$ their final continuum state with momentum $\kappa_{\mathrm{T}}$ and $\Delta E_{f n}^{\mathrm{T}}$ their energy transfer. Excitation to bound target states has been neglected because ionization is the dominant process (Day 1981). Likewise, the ionization probability for core electrons is small, hence the sum over $n$ can be restricted to the target valence electrons. In equation (4) $f_{\mathrm{e}}$ is the elastic scattering amplitude (with $\left|f_{\mathrm{e}}\right|^{2}=\mathrm{d} \sigma_{\mathrm{e}} / \mathrm{d} \Omega$ ), and the on-shell prescription is the same as given below equation (1).

In order to test the theoretical models, we have calculated electron loss for $0.4 \mathrm{MeV} \mathrm{amu}{ }^{-1} \mathrm{He}^{+}$on Ar at $\vartheta_{j}=150^{\circ}$ (figure 1) where experimental data are available (DuBois and Manson 1990). The EIA and the peaked SB2 calculations for the singly inelastic (sr) contribution were performed using equations (1) and (3), respectively, without additional peaking approximations. The elastic scattering amplitude entering into these equations was obtained from a phaseshift analysis of the solutions of the radial Schrödinger equation for electron scattering by the target field (Kuzel et al 1992). Twenty partial waves had to be included. Clearly, the si contribution alone cannot account for the experimental intensities except possibly at the highest energies. On the other hand, upon adding the doubly inelastic contribution obtained from equation (4) with the help of additional peaking approximations as discussed in earlier 


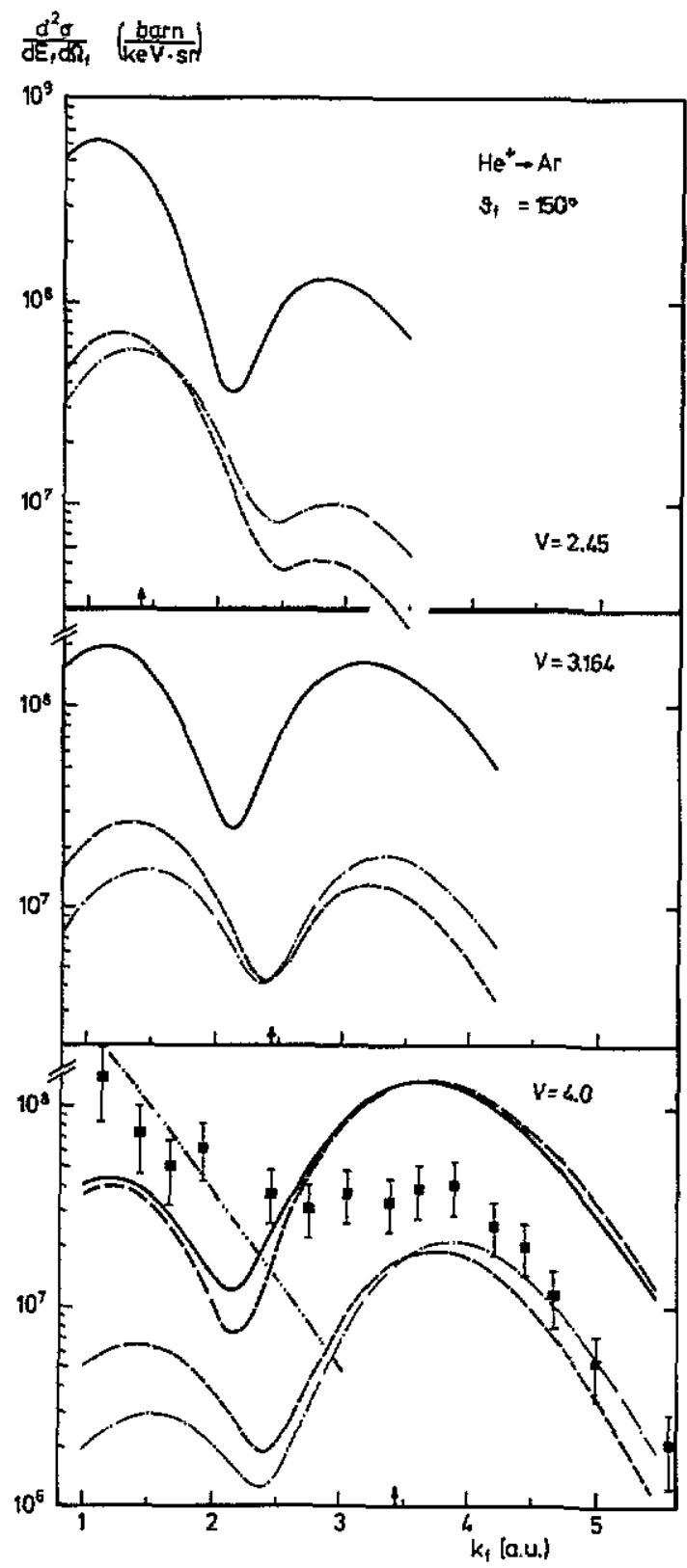

Figure 1. Doubly differential electron loss cross section in $0.15 \mathrm{MeV} \mathrm{amu}^{-1}(v=2.45 \mathrm{au})$, $0.25 \mathrm{MeVamu}^{-1}(v=3.164 \mathrm{au})$ and $0.4 \mathrm{MeVamu}^{-1}(v=4 \mathrm{au}) \mathrm{He}^{+}+\mathrm{Ar}$ collisions at $\vartheta_{f}=$ $150^{\circ}$. The experimental data are from DuBois and Manson (1990). Theory: - - , EIA (singly inelastic contribution, equation (1)); - - - peaked SB2 ( $S$ I contribution, equation (3)); - EIA plus DI contribution from equation (4); - - , EIA plus modified DI contribution from equation (4) with (5); - - c contribution from target electrons. The arrow marks $\frac{1}{2} k_{f}^{2}=E_{\text {loss }}=\frac{1}{2} v^{2}+\varepsilon_{i}^{P}$. 
work (Kuzel et al 1992, Jakubassa-Amundsen 1992), the data are overestimated at the higher energies. One might expect that this could be caused by the breakdown of a first Born type treatment of target ionization when the perturber charge is larger than the effective charge of the valence electrons. Therefore we have tentatively accounted for the interaction of the target electron with the projectile field to infinite order by replacing in (4) the first Born approximation to the scattering amplitude with the exact Coulomb scattering amplitude

$$
f^{\mathrm{B} 1}(s)=\frac{2 Z_{\mathrm{P}}}{s^{2}} \rightarrow f^{\text {Coul }}(K, s)=\frac{2 Z_{\mathrm{P}}}{s^{2}} \frac{\Gamma(1-\mathrm{i} \eta)}{\Gamma(1+\mathrm{i} \eta)} \exp [2 \mathrm{i} \eta \ln (s / 2 K)]
$$

where $\eta=Z_{\mathrm{P}} / K, s$ is the momentum transfer, and $K=\max \left(\left|\kappa_{\mathrm{T}}-\boldsymbol{v}\right|,\left|\kappa_{\mathrm{T}}-\boldsymbol{v}-\boldsymbol{s}\right|\right)$ in the on-shell approximation. We have found that this modified prescription for DI leads only to minor modifications of the electron loss cross section. Hence, the discrepancies with experiment for $k_{f} \geqslant 3$ au are probably due to the peaking approximations applied to (4) which are less valid, the more tightly the projectile electron is bound. Because of limited computer time, we did not find it possible to relax these additional approximations.

The deviations between theory and experiment at low energies are attributed to the contribution of target electrons to the experimental yield (in addition to the projectile electrons) which are recorded simultaneously with charge-changed projectiles (Maier 1992). In order to estimate the magnitude of this target-electron contribution, we have used (4) but integrated over $\boldsymbol{k}_{f}$ instead of $\boldsymbol{\kappa}_{\mathrm{T}}$. Due to the approximation of the target valence states by scaled 1s hydrogen-like functions, this method is not very accurate, but it confirms the conjecture (figure 1).

Hence, the qualitative energy dependence of the experimental data can be explained by adding to the SI (either EIA or peaked SB2) plus Dr contribution the contribution from the target electrons. We note that the ErA prescription for $\mathrm{sx}$ is somewhat superior to the peaked SB2. This is expected from the concept that, at backward emission angles, the ejected electron is predominantly influenced by the target field.

Having established the validity of our theoretical approach, we turn to the investigation of the loss peak structures. When the collision velocity is lowered (but the emission angle kept fixed at $150^{\circ}$, figure 1), the peak near $E_{\text {loss }}$ which exists for high velocities decreases, and a second peak at lower energies gains increasing importance. At very low energies, the location of this second peak coincides again with $E_{\text {loss }}$. Hence, the position of the electron loss peak does not shift proportional to $\frac{1}{2} v^{2}$ as predicted by the first Born theory.

At intermediate velocities, $v \sim 3$ au, the two peaks are of nearly equal magnitude, and a minimum appears near $E_{\text {loss. }}$. In figure 2 the angular dependence of this structure is investigated at $0.25 \mathrm{MeV} \mathrm{amu}^{-1}$, and similar features are found as if the velocity were changed. At angles below $120^{\circ}$, there is only one peak near the energy $E_{\text {loss }}$. With increasing $\vartheta_{f}$, this peak shifts to lower energies while a second peak at higher energies emerges. The two maxima in the electron loss peak region are present for all emission angles $120^{\circ} \leqslant \vartheta_{f} \leqslant 180^{\circ}$, with the minimum located at $E_{\text {loss }}$ for $\vartheta_{f} \approx 130^{\circ}$.

In order to trace the origin of this behaviour, one has to recall that at backward angles, the influence of the weak projectile core field on the electron is negligible, once it is ejected. Therefore, the angular distribution of the emitted electrons will be closely related to the differential cross section for free electrons scattered by the target potential. The inelasticity of the electron loss process allows for a probing of the energy variations of this differential cross section. The probed energy region is larger, the heavier the 


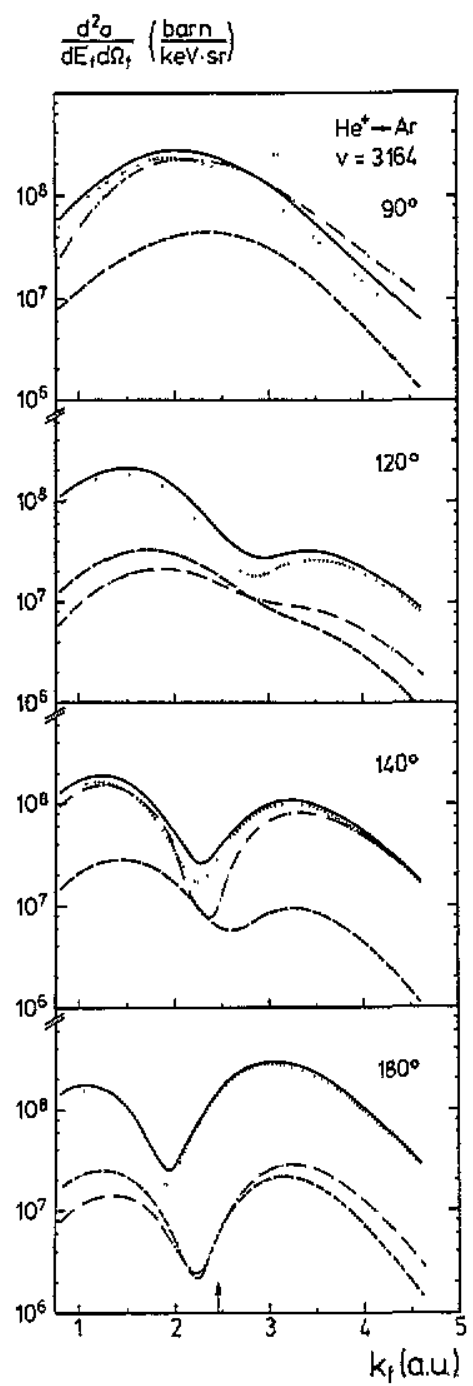

Figure 2. Doubly differential electron loss cross section in $0.25 \mathrm{MeV} \mathrm{amu}^{-1} \mathrm{He}^{+}+\mathrm{Ar}$ collisions as function of electron momentum $k_{f}$ at emission angles $\vartheta_{f}=90^{\circ}, 120^{\circ}, 140^{\circ}$ and $180^{\circ}$. - - -, EIA (SI contribution); - - - peaked SB2 (si contribution); $\cdots$, DI contribution; $-\cdots-$, modified DI contribution; — , sum of EIA and DI contribution from equation (4). The arrow marks $E_{\text {loss }}$

projectile is (i.e. the larger the intrinsic momentum components of the bound-state function), and the more the electron momentum $k$, differs from the average momentum $v$ of a projectile electron (in the target rest frame). Likewise, the inelasticity of the loss process leads to a difference between the scattering angle $\theta$ and the emission angle $\vartheta_{f}$ (cf the discussion below equation (1)) such that also the angular variations of the elastic electron scattering cross section are probed. Hence, when there is a strong energy or angular variation of $\mathrm{d} \sigma_{\mathrm{e}} / \mathrm{d} \Omega(k, \theta)$ at certain $k$ and $\theta$, this is likely to show up in the electron loss spectrum recorded at an angle in the vicinity of this particular angle $\theta$ and at an impact velocity close to that $k$. We attribute the minimum in the 
electron loss peak region for backward angles to the pronounced Ramsauer-Townsend minimum near $\theta=120^{\circ}$ in the elastic electron-argon scattering cross section (McCarthy et al 1977). Likewise, the change in peak height with varying $\vartheta_{f}$ can be related to the strong energy variation of this cross section near $k=3 \mathrm{au}$.

Since both si theories as well as the DI theory (via the treatment of the projectile electron ejection) are dominated by the influence of the elastic electron scattering amplitude or scattering cross section, all the contributions to electron loss show the structures in a similar way. The modified DI prescription ((4) with (5)) deviates more from the first-order treatment of target excitation when the collision energy is lowered (figure 2), the structures being even more pronounced. We have tested the influence of the target atomic potential on the loss peak structures by excluding shell effects, i.e. by replacing the Hartree-Fock potential by an exponentially screened Coulomb field (with the Thomas-Fermi screening constant) in the Schrödinger equation for elastic electron scattering. Even then, two peaks appear, but they have different intensity ratios and their energy positions are shifted. This indicates that the particular shape of the structures is sensitive to details of the target field, but that their origin (or rather the origin of the Ramsauer-Townsend minima) is due to the interference of the various partial waves contributing to $f_{\mathrm{e}}$.

In order to investigate the dependence on the projectile charge, we show in figure 3 electron loss intensities in $\mathrm{H}+\mathrm{Ar}$ collisions at $\vartheta_{f}=150^{\circ}$. The experimental data (Kuzel et al 1992) for $0.5 \mathrm{MeV}$ projectiles are in the peak region well described by the singly inelastic contribution (again, EIA gives the better results), while the Di contribution is rather small. This improved agreement as compared to previous calculations for this system (Kuzel et al 1992) is due to the omission of the transverse peaking in the singly inelastic contribution (1) and the allowance for variations of the scattering angle $\left(\theta \neq \vartheta_{f}\right)$, but most importantly, the omission of the closure approximation in the DI contribution (which becomes invalid for large momentum transfers in the case of heavy targets). For energies below the electron loss peak, the simultaneous projectiletarget excitation is the dominant process, but because of the less tightly bound projectile electron not so important as for $\mathrm{He}^{+}$. Again, the large experimental intensities at low energies may be explained by means of the additionally recorded target electrons.

When the collision velocity is lowered to $0.25 \mathrm{MeV}$, the electron loss peak is still close to $E_{\text {loss, }}$, and the second peak at lower energies is one order of magnitude less intense, in contrast to the results for the heavier $\mathrm{He}^{+}$projectile. The reason for the weakly developed loss peak structures for hydrogen impact lies in the fact that the narrow initial-state momentum distribution of the projectile electron (as compared to $\mathrm{He}^{+}$) does not allow for a sufficient probing of the strong variations of the elastic scattering amplitude with energy and angle.

For displaying the effect of peaking approximations on the structure, we have included in figure 3 the result from the unpeaked SB2 theory (Jakubassa-Amundsen 1993). Clearly, the existence of the structure is not affected when the peaking approximation is dropped. However, for the case of backward emission, the unpeaked SB2 seems to give poorer results (as compared to the experimental trend) than the peaked SB2 because the properties of the target continuum eigenstate (which in SB2 appears only as an intermediate state) are veiled more by the extra integration.

We have also made calculations for $\mathrm{He}^{+}$colliding with $\mathrm{Xe}$, and found a double peak structure which is very much like the one for Ar targets, and which occurs in the same angular and velocity range. Again, it can be traced back to a Ramsauer-Townsend minimum near $\theta=145^{\circ}$ for elastic electron scattering on Xe (McCarthy et al 1977). 


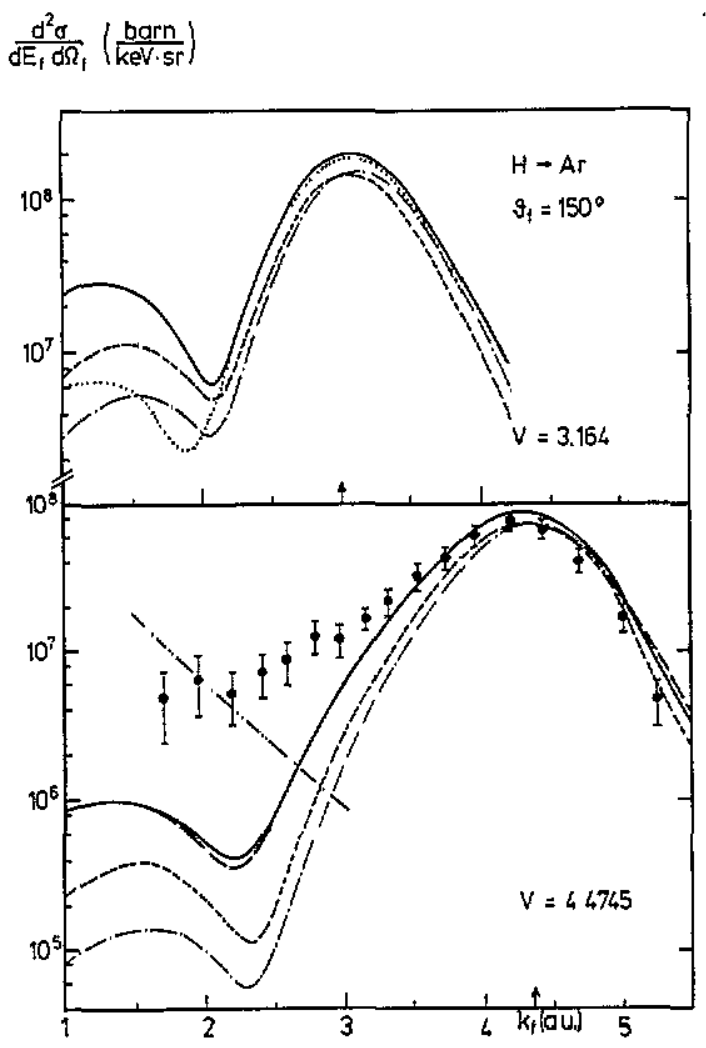

Figure 3. Doubly differential electron loss cross section in $0.25 \mathrm{MeV}(v=3.164 \mathrm{au})$ and $0.5 \mathrm{MeV}(v=4.4745 \mathrm{au}) \mathrm{H}+\mathrm{Ar}$ collisions at $\vartheta_{f}=150^{\circ}$. The experimental data are from Kuzel et al (1992). The error bars do not include the absolute uncertainty. Theory: - - -, $E I A,-\cdot-$, peaked $S B 2, \cdots$, unpeaked SB2, all S1 contribution; - - ElA plus DI contribution; - - EIA plus modified DI contribution; - $\cdot-$, contribution from target electrons. The arrow marks $E_{\text {loss }}$.

In conclusion, theoretical evidence has been given for the existence of a double peak structure in the electron loss spectra for emission angles $120^{\circ} \leqslant \vartheta_{f} \leqslant 180^{\circ}$, which is most pronounced for $\mathrm{He}^{+}$impact at a collision velocity near 3 au. The origin of the loss peak structure is the same as underlies the binary encounter peak structures in the reversed collision systems (Hagmann et al 1992): in an energetic ion-atom encounter, the loosely bound electrons which are ejected into the vicinity of the heavy collision partner, behave as quasifree particles in the field of this atom (or ion). Hence, the spectral distribution of the electrons mirrors the diffraction pattern of the corresponding elastic electron scattering cross section. The loss peak structures are present for all heavy targets, but the detailed form depends on the target species. An experimental investigation of such collision systems down to $v \leqslant 3$ au is clearly desirable, preferably by recording the charge state of the recoiling target in coincidence. Experiments of this type should be feasible nowadays (Montenegro et al 1992).

It is a pleasure to thank $\mathrm{H}$ Schmidt-Böcking and $\mathrm{MW}$ Luvas for many stimulating discussions and for access to their data prior to publication. Support from the GSI Darmstadt is gratefully acknowledged. 


\section{References}

Briggs J S and Drepper F 1978 J. Phys. B: At. Mol. Phys. 114033

Burch D, Wieman H and Ingalls W B 1973 Phys. Rev. Lett. 30823

Day M H 1981 J. Phys. B: At. Mol. Phys. 14231

Drepper F and Briggs J S 1976 J. Phys. B: At. Mol. Phys. 92063

DuBois R D and Manson S T 1986 Phys. Rev. Lett. 571130

1990 Phys. Rev. A 421222

Hagmann S et al 1992 J. Phys. B: At. Mol. Opt. Phys. 25 L287

Hartley H M and Walters H R J 1987 J. Phys, B: At. Mol. Phys. 203811

Jakubassa D H 1980 J. Phys. B: At. Mol. Phys. 132099

Jakubassa-Amundsen D H 1992 Z. Phys. D 22701

1993 submitted for publication

Jakubassa-Amundsen D H and Amundsen P A 1980 Z. Phys. A 297203

Kelbch C, Hagmann S, Kelbch S, Mann R, Olson R E, Schmidt S and Schmidt-Böcking H 1989 Phys. Lett. 139A 304

Kuzel M, Heil O, Maier R, Lucas M W, Jakubassa-Amundsen D H, Farmery B W and Groeneveld K O 1992 J. Phys. B: At. Mol. Opt. Phys. 251839

Macek J H and Shakeshaft R 1980 Phys. Rev. A 221441

Maier R 1992 Private communication

McCarthy I E, Noble C J, Phillips B A and Turnbull A D 1977 Phys. Rev. A 152173

Montenegro E C, Melo W S, Meyerhof WE and de Pinho A G 1992 Phys. Rev. Lett. 693033

Reinhold C O, Schultz D R, Olson R E, Kelbch C, Koch R and Schmidt-Böcking H 1991 Phys. Rev. Lett. 661842

Richard P, Lee D H, Zouros T J M, Sanders J M and Shinpaugh J L 1990 J. Phys. B: At. Mol. Opt. Phys. 23 L213

Shingal R, Chen Z, Karim K R, Lin C D and Bhalla C P 1990 J. Phys. B: At. Mol. Opt. Phy's. 23 L637

Walters H R J 1975 J. Phys. B: At. Mol. Phys. 8 L54

Wang J, Reinhold C O and Burgdörfer J 1992 Phys. Rev. A 45450 ? 